\title{
Identification of a chrXq27.3 microRNA cluster associated with early relapse in advanced stage ovarian cancer patients
}

\section{Marina Bagnoli, Loris De Cecco, Anna Granata, Roberta Nicoletti, Edoardo Marchesi, Paola Alberti, Barbara Valeri, Massimo Libra, Mattia Barbareschi, Francesco Raspagliesi, Delia Mezzanzanica and Silvana Canevari \\ Oncotarget $2011 ; 2: 1265-1278$.}

\section{Addendum}

We submitted to GEO repository new information to be added to the SuperSeries GSE25204 including: a new dataset, GSE67819, reporting the microRNA microarray profiling of our validation set; an update of the characteristic files including clinical data of patients in the GSE25202, GSE25203 and GSE67819 datasets. 